model 402 accounting machine, and the sum checks

$$
\sum_{m} A_{m}(n) \stackrel{?}{=} 1
$$

were totalled and listed concurrently. Inspecting the sum checks and spot-checking by hand-computation suggest that the results $A_{m}(n)$ are accurate to 14 decimals.

The $A_{m}(n)$ were rounded on cards to 15 decimals, using an International Business Machines Corporation model 075 sorter for machine inspection of the 16th decimal, and an International Business Machines Corporation model 513 reproducer for subsequent insertion of the corrected 15 th decimal. It is believed that the error in $A_{m}(n)$ is $\leq 1 \times 10^{-15}$.

University of California

R. B. Horgan

Los Angeles, California

1. Herbert E. Salzer, "Formulas for the partial summation of series" [see p. 149 this issue].

\title{
Polynomial Approximations to Some Modified Bessel Functions
}

The following approximations were determined by equating tabular values of the functions to polynomials of degree $n$ at $n+1$ points and solving the equations for the $n+1$ coefficients. In the region from 0 to $x_{r}$, the fit points were spaced at equal intervals in $x^{2}$; in the region from $x_{r}$ to $\infty$, they were spaced at equal intervals in $1 / x$. Both $n$ and $x_{r}$ were determined by trial and error with the following requirements.

(1) To yield 7 or 8 accurate significant figures over the full range of positive $x$.

(2) To make the polynomial degrees approximately the same in the two regions 0 to $x_{r}$ and $x_{r}$ to $\infty$.

(3) To keep the magnitudes of the polynomial coefficients, the definitions of the polynomials, and the regions of validity balanced to the extent that a given number of significant figures in the coefficients would give the same number of significant figures in the polynomials over the entire region of validity.

In order to determine the maximum errors, the polynomials were evaluated and compared (directly or after appropriate multiplications and additions) with values given in [1]. Differences between calculated and tabular values were plotted for

$$
x=.0[.1] 5[.2] 7.6[.4] 10[1] 20 .
$$

To determine maximum error estimates in formulas (3) and (4), it was necessary to compute $I_{0}$ and $I_{1}$ at higher values of $x$ from asymptotic expansions. Local maxima in these two formulas occur approximately at midpoints between fit points, decreasing gradually as $x$ is decreased. The lowest is about $.1 \times 10^{-7}$ at $x=4.1$ and the highest, about $2 \times 10^{-7}$ at $x=60$.

These approximations may be considered a sequel to a similar set for $J_{0}, Y_{0}$, $J_{1}$, and $Y_{1}$, which was published earlier [2]. 
(1) $-3.75 \leq x \leq 3.75 \quad|\epsilon|_{\max } \sim 3.0 \times 10^{-8}$

$$
\begin{aligned}
I_{0}(x) \simeq 1 & +3.5156229(x / 3.75)^{2}+3.0899424(x / 3.75)^{4} \\
& +1.2067492(x / 3.75)^{6}+.2659732(x / 3.75)^{8} \\
& +.0360768(x / 3.75)^{10}+.0045813(x / 3.75)^{12}
\end{aligned}
$$

(2) $-3.75 \leq x \leq 3.75$

$$
|\epsilon|_{\max } \sim 1.0 \times 10^{-8}
$$

$$
\begin{aligned}
I_{1}(x) / x \simeq .5 & +.87890594(x / 3.75)^{2}+.51498869(x / 3.75)^{4} \\
& +.15084934(x / 3.75)^{6}+.02658733(x / 3.75)^{8} \\
& +.00301532(x / 3.75)^{10}+.00032411(x / 3.75)^{12}
\end{aligned}
$$

(3) $3.75 \leq x<\infty$

$$
|\epsilon|_{\max } \sim 2 \times 10^{-7}
$$

$$
\begin{aligned}
& I_{0}(x) x^{\frac{1}{2}} e^{-x} \simeq .398942280 \quad+.013285917(3.75 / x) \\
& +.002253187(3.75 / x)^{2}-.001575649(3.75 / x)^{3} \\
& +.009162808(3.75 / x)^{4}-.020577063(3.75 / x)^{5} \\
& +.026355372(3.75 / x)^{6}-.016476329(3.75 / x)^{7} \\
& +.003923767(3.75 / x)^{8}
\end{aligned}
$$

(4) $3.75 \leq x<\infty$

$$
|\epsilon|_{\max } \sim 2 \times 10^{-7}
$$

$$
\begin{aligned}
& I_{1}(x) x^{\frac{1}{3}} e^{-x} \simeq \quad .398942280 \quad-.039880242(3.75 / x) \\
& -.003620183(3.75 / x)^{2}+.001638014(3.75 / x)^{3} \\
& -.010315550(3.75 / x)^{4}+.022829673(3.75 / x)^{5} \\
& -.028953121(3.75 / x)^{6}+.017876535(3.75 / x)^{7} \\
& -.004200587(3.75 / x)^{8}
\end{aligned}
$$

(5) $0<x \leq 2$

$$
|\epsilon|_{\max } \sim 7.0 \times 10^{-8}
$$

$$
\begin{aligned}
K_{0}(x)+\ln (.5 x) I_{0}(x) \simeq & -.57721566 \quad+.42278420(x / 2)^{2} \\
& +.23069756(x / 2)^{4}+.03488590(x / 2)^{6} \\
& +.00262698(x / 2)^{8}+.00010750(x / 2)^{10} \\
& +.00000740(x / 2)^{12}
\end{aligned}
$$

(6) $0<x \leq 2$

$$
|\epsilon|_{\max } \sim 6.0 \times 10^{-8}
$$

$$
\begin{aligned}
& \left\{K_{1}(x)-\ln (.5 x) I_{1}(x)\right\} x \simeq 1+.15443144(x / 2)^{2} \\
& \text { - } .67278579(x / 2)^{4}-.18156897(x / 2)^{6} \\
& -.01919402(x / 2)^{8}-.00110404(x / 2)^{10} \\
& -.00004686(x / 2)^{12}
\end{aligned}
$$

(7) $2 \leq x<\infty$

$$
|\epsilon|_{\max } \sim 1.5 \times 10^{-7}
$$

$$
\begin{aligned}
K_{0}(x) x^{\frac{1}{1}} e^{x} \simeq & 1.25331414-.07832358(2 / x) \\
& +.02189568(2 / x)^{2}-.01062446(2 / x)^{3} \\
& +.00587872(2 / x)^{4}-.00251540(2 / x)^{5} \\
& +.00053208(2 / x)^{6}
\end{aligned}
$$

(8) $2 \leq x<\infty$

$$
|\epsilon|_{\max } \sim 1.5 \times 10^{-7}
$$

$$
\begin{aligned}
K_{1}(x) x^{\frac{1}{3}} e^{x} \simeq & 1.25331414 \quad+.23498619(2 / x) \\
& -.03655620(2 / x)^{2}+.01504268(2 / x)^{3} \\
& -.00780353(2 / x)^{4}+.00325614(2 / x)^{5} \\
& -.00068245(2 / x)^{6}
\end{aligned}
$$


In these formulas, $\epsilon=\frac{\text { calculated } F-\operatorname{tabular} F}{\text { tabular } F}$ where $F=I_{0}, I_{1}, K_{0}$, or $K_{1}$. [ $\epsilon$ given in the approximations to $J_{0}, Y_{0}, J_{1}$, and $Y_{1}$ previously mentioned was the absolute error in the functions represented by polynomials.]

Shell Development Company

E. E. Allen

Exploration and Production Research Division

Houston, Texas

1. British Association for the Advancement of Science, Mathematical Tables, v. X, Bessel Functions, Cambridge Univ. Press, 1952.

2. E. E. AlLEN, “Analytical approximations," $M T A C$, v. 8, 1954, p. 240-241.

$$
\begin{aligned}
& \text { Radix Tables for } \sin x \text { and } \cos x, x=a \cdot 10^{k} \text { degrees, } \\
& \qquad a=1(1) 9, k=-3(1) 1
\end{aligned}
$$

These tables permit calculation of $\sin x$ or $\cos x$ for any argument $x$ expressed to $3 \mathrm{D}$ by means of summation formulas listed in Table $\mathrm{I}$ and requiring at most 64 multiplications and 15 additions or subtractions.

\section{No. of decimals in $x$}

3

2

1

0

\section{Maximum number of multiplications}

64

24

8

2

\section{Maximum number of additions or subtractions}

The table was computed on the SWAC machine using double precision routines. (Each SWAC number has a sign plus 36 binary digits.) Values of $1 / n$ ! for $n=2(1) 20$ were taken from Peters and Stein [1].

For $x=.001(.001) .01(.01) .1(.1) 1(1) 10(10) 50^{\circ}$, expressed in radians, double precision, the routine computed $\sin x$ and $\cos x$ by

$$
\sin x=x-\frac{x^{3}}{3 !}+\frac{x^{5}}{5 !}-\frac{x^{7}}{7 !}+\cdots-\frac{x^{19}}{19 !}
$$

and

$$
\cos x=1-\frac{x^{2}}{2 !}+\frac{x^{4}}{4 !}-\frac{x^{6}}{6 !}+\cdots+\frac{x^{20}}{20 !}
$$

For each $x$, as a term $\frac{x^{m}}{m !}$ was computed, it was accumulated into the $\sin x$ or the $\cos x$ cell (alternating) with the proper sign attached. The limit of 20 was chosen for $n$ in the formulas above, since there would be no contribution from succeeding 